\title{
La lucha de los profesores por la disciplina escolar: una mirada desde la histórica
}

\section{The teachers' struggle for school discipline: a historical perspective}

\author{
DOI: $10.46932 / \mathrm{sfjdv3n2-002}$
}

Received in: February $28^{\text {th }}, 2022$

Accepted in: March $1^{\text {st }}, 2022$

\section{María Ana Valle Barbosa \\ Dra}

Profesor Investigador titular B, miembro del C. A. Ciencias Sociales y Salud (071) Línea de Investigación Procesos Históricos y Salud

Institución: Centro Universitario de Ciencias de la Salud, Universidad de Guadalajara, Guadalajara Jalisco, México

Dirección: Sierra Mojada No. 950, Col. Independencia C.P. 44348, Guadalajara, Jalisco, México

México, Guadalajara

Correo electrónico: mavalleb@yahoo.com.mx

\section{María Elena Flores Villavicencio}

Dra

Profesor Investigador titular C, miembro del C. A. Ciencias Sociales y Salud (071) Línea de Investigación Psicología Social y Salud y miembro del Sistema Nacional de investigadores nivel I Institución: Centro Universitario de Ciencias de la Salud, Universidad de Guadalajara Guadalajara Jalisco, México

Dirección: Sierra Mojada No. 950, Col. Independencia C.P. 44348, Guadalajara, Jalisco, México México, Guadalajara

\section{María Guadalupe Vega López \\ Dra}

Profesor Investigador titular C, miembro del C. A. Salud y Población (068) Línea de Investigación

Salud y desarrollo Humano y miembro del Sistema Nacional de investigadores nivel II.

Institución: Centro Universitario de Ciencias de la Salud, Universidad de Guadalajara, Guadalajara Jalisco, México

Dirección: Sierra Mojada No. 950, Col. Independencia C.P. 44348, Guadalajara, Jalisco, México México, Guadalajara

\section{Armando Muñoz de la Torre Dra., MCSP}

Profesor Investigador titular B, miembro del C. A. Ciencias Sociales y Salud (071) Línea de Investigación Procesos Históricos y Salud

Institución: Centro Universitario de Ciencias de la Salud, Universidad de Guadalajara, Guadalajara Jalisco, México

Dirección: Sierra Mojada No. 950, Col. Independencia C.P. 44348, Guadalajara, Jalisco, México México, Guadalajara

\section{RESUMEN}

Objetivo: Describir la importancia de la disciplina escolar en la aulas, asimismo, a la problemática que se enfrentan los profesores para implementarla. Históricamente la disciplina en las escuelas se lograba mediante castigos, y Guadalajara, México, no fue la excepción. En la reglamentación de la Escuela de 
Artes, Oficios y Primeras Letras de Guadalajara los castigos han dependían de la observación del educador. Este fenómeno no es una problemática reciente, desde la revolución industrial se vigilaron los derechos de los niños y niñas, de acuerdo con las condiciones de abuso y explotación laboral que enfrentaban en dicho momento histórico. Hoy son temas de interés para los derechos humanos y la Salud Pública. En la actualidad, tanto el gobierno como la sociedad civil se han preocupado por la socialización escolar poniendo en práctica programas de intervención y prevención para impedir actos de insubordinación escolar, al mismo tiempo, sin dejar de lado los derechos de alumnos y profesores, considerarse de manera conjunta para conseguir la armonía necesaria del buen funcionamiento de las instituciones educativas, dicho de otra manera, que haya paciencia para admitir otras opiniones de las personas con respeto a su dignidad.

Palabras clave: disciplina, castigos, niños, maestros, derechos humanos.

\begin{abstract}
Objective: To describe the importance of school discipline in the classroom, as well as the problems faced by teachers in implementing it. Historically, discipline in schools was achieved through punishment, and Guadalajara, Mexico, was no exception. In the regulations of the Escuela de Artes, Oficios y Primeras Letras de Guadalajara, punishments have depended on the observation of the educator. This phenomenon is not a recent problem; since the industrial revolution, the rights of children have been monitored, in accordance with the conditions of abuse and labor exploitation they faced in that historical moment. Today they are issues of interest for human rights and public health. At present, both the government and civil society have been concerned about school socialization by implementing intervention and prevention programs to prevent acts of school insubordination, at the same time, without leaving aside the rights of students and teachers, to be considered together to achieve the necessary harmony of the proper functioning of educational institutions, in other words, that there is patience to admit other opinions of people with respect for their dignity.
\end{abstract}

Keywords: discipline, punishments, children, teachers, human rights.

\title{
1 INTRODUCCIÓN
}

El salón de clases en la actualidad es un espacio donde se han perdido valores éticos y morales, los alumnos saludan a los docentes con un insulto y no pasa nada, y en esas condiciones desempeñar la tarea el profesor es un reto. Como en cualquier otro espacio es necesario que haya normas para lograr una buena calidad educativa, de trasgredir las reglas se emplearan sanciones llamadas coloquialmente castigos, los cuales tienen que ajustarse a las leyes y derechos que protegen al menor. Sin embargo, para el maestro desempeñar la misión de educar sin disciplina escolar es una gestión casi imposible, ahí radica la importancia apoyo de los padres o tutores con sus hijos en las tareas del maestro, además, reconsiderar las leyes que planteen la posibilidad de la aplicación de medidas correctivas objetivas a los niños, niñas y adolescentes por parte de los mentores. 


\section{METODOLOGÍA}

Este trabajo presenta un avance de resultados de la investigación titulada "Historia de la violencia escolar en Guadalajara, Jalisco, México”. El objetivo de este documento es describir la importancia de la disciplina escolar en las aulas, los derechos de los niños, igualmente, la importancia de la falta de protección a los docentes, ya que, fuera de la institución se sientes amenazados por los propios alumnos cuando se implementan medidas disciplinarias en las aulas para desarrollar el docente los objetivos del curso.

Para la elaboración de este artículo, se utilizó la metodología histórica, la cual se define por la manera de enfocar los problemas y encontrar respuestas del presente respecto al pasado, que surge como una representación de sí mismo que puede lograrse a partir del acto de escribir, esto implica rescatar el sentido de los antecedentes históricos para retornar una vez más hacia el presente. Es la forma de comprender lo actual, el pasado y lo futuro (Tovar, 2014).

\subsection{LA DISCIPLINA ESCOLAR}

Para hablar de la indisciplina, cabe exponer que la disciplina es un conjunto de precedentes para segura el orden y convivencia en la escuela y evitar que se cometan atropellos o se apliquen criterios personales, según las simpatías o antipatías, y el estado de animo dela autoridad (Treviño y González, 2020).

Por lo tanto, En todas las instituciones educativas es necesario una normatividad interna, la cual debe ser acatada por sus integrantes para que haya orden en el desarrollo de los trabajos correspondientes. De lo contrario, las asignaturas no se podrán impartir. Otra tarea del profesor en turno es poner en práctica la disciplina para lograr sus objetivos. por ello, la educación no solo es conocimiento, sino también contribuir a la formación integral del estudiante (Treviño y González, 2020)

Por consiguiente en los planteles magistrales y principalmente en el aula donde el profesor interactúa con los alumnos, el docente tiene que mantener al grupo organizado para reducir la indisciplina y la agresividad. Por lo tanto, el orden es necesario dentro del grupo. Dicho de otra manera, debe aplicarse un conjunto de normas que regulen la convivencia en las aulas, refiriéndose tanto al orden colectivo como a las prácticas de organización y respeto entre cada uno de los miembros que constituyen la colectividad educativa (García, 2008). Por consiguiente, la disciplina tiene un papel importante, ya que, ésta ayudará a un mejor aprovechamiento del tiempo, para realizar el trabajo integrador del alumno. En este sentido, la disciplina es entendida como el conjunto de acciones cuyas estrategias permitirán llevar una convivencia segura de todos los miembros del grupo, asimismo, como el conjunto de acciones desarrolladas por el profesor, orientadas al escolar para enrole en los trabajos que se desarrollen en el salón de clase y para 
minimizarán los comportamientos disruptivos en el grupo (Márquez, 2007). En otras palabras, es el conjunto de actividades planificadas y destinadas a conseguir el orden en el salón de clases.

Tanto para a los profesores, padres de familia y alumnos les debe quedar claro que, el objetivo de la disciplina no consiste en la aplicación de la autoridad sobre los alumnos, sino facilitar el desarrollo del proceso enseñanza-aprendizaje (García, 2008). Sobre todo en las clases la disciplina se ejerce como medio de apoyo para realizar con mejor calidad las metas, así se logrará una mejor coordinación del grupo, por consiguiente, los alumnos conseguirán una socialización más integral. Cabe señalar que, por un lado, la disciplina se entiende como correctiva de conductas inadecuadas y, por otra parte, como estrategia para tener el control de las conductas no deseadas.

\subsection{INDISCIPLINA ESCOLAR}

Si no se tienen un orden y convivencia en el proceso enseñanza-aprendizaje, se pude deducir que se está ante episodio de indisciplina escolar, entendida como una conducta disruptiva que no promueve la armonía y la convivencia en la sociedad, especialmente entre los escolares es lamentable que la indisciplina no se haya podido combatir por un sinnúmero de factores que no ayudan a erradicarla (Ponce, Quiñónez y Gómez, 2020).

En el mismo orden de ideas, García (2008), destacó que, el fenómeno de la indisciplina es una situación que preocupa tanto al profesorado como a los responsables de la Administración Educativa. La inquietud surge por la periodicidad con que se viven eventos que alteran y desequilibran la convivencia de los alumnos en el aula, además, refirió que hay dificultad en identificar soluciones idóneas y eficaces para superar el problema de la indisciplina.

De ahí que, al presente se escriben y se publican numerosos artículos en gran parte del mundo que manifiestan los problemas de indisciplina en las escuelas, y de la interacción del profesor-alumno (Suarez, 2004. Viana, 2010 y Furlán 2005). También, de lo complejo que resulta desempeñar las funciones como docentes en un ambiente de rebeldía, el cual no es propio para el proceso formativo del alumno.

Márquez (2007) describió que primitivamente, la disciplina en las escuelas se lograba infundiendo miedo a los alumnos a través de los castigos, practicándose más la sanción física, la cual llegó a ser muy difundido en el continente americano, al punto que se constituyó una hábito aceptados socialmente por la mayor parte del mundo como una forma de disciplina (Paima, 2009).

Esa aceptación contribuyó para que un acto de indisciplina del alumno se hiciera acreedor a una sanción, siendo el más usual el castigo corporal como palizas, pellizcos, cachetadas, tirones de pelo y de orejas, coscorrones, azotes etc., en algunas ocasiones el maestro utilizaba sus propias manos y en otras instrumentos de castigo: regla, punteros, varas, correas de cuero, etc., ultrajando así todo el cuerpo del 
escolar. La finalidad de los correctivos se ha manipulado en dos dimensiones, uno relacionada con el rendimiento académico y la otro con el comportamiento del alumno (Suárez, 2004).

Históricamente, los correctivos han sido una estrategia para regular el comportamiento de los educandos en el aula, los cuales han cambiado de acuerdo al tiempo y espacio, por ejemplo: en Egipto era memorización y azotes, en Roma al principio de nuestra era azotes; actividad criticada por Marco Fabio Quintiliano, en la escuela hispano musulmán se avalaron las sanciones físicas con cañas, varas, reglas, cuerdas, latiguillos o cinturones por los padres de familia. En el Cairo más recientemente las lecciones se hacían con correa en mano, llevándose puntualmente, la letra con sangre entra y la labor con dolor. En Marruecos, el maestro manipulaba un puntero para pinchar a los alumnos por la espalda. Ante estos escenarios, Lutero y Jhon Lock en el siglo XVII, y Rousseau en el siglo XVIII pugnan por una instrucción más afable para los niños. Mientras que en España, Alberto Lista justificaba los castigos como necesarios para frenar la inmoralidad, la falta de desempeño y la indisciplina perpetuada, y las puniciones corporales sólo podían ser practicadas por los padres. En Europa occidental surgen iniciativas humanistas, en la segunda mitad del siglo XIX y mediados del XX, Tolstoy y Montessori impulsan una nueva educación donde el castigo queda relegado al menos sobre el papel. A pesar de, en España en el siglo XX continuaban las prácticas anteriores, los niños eran obligados a realizar trabajos forzados ocasionalmente, eran castigados de acuerdo a la gravedad del delito y así, muchas voces se alzaron por la humanización de la enseñanza (Navarro, 2004).

Al presente, en Guadalajara, Jalisco, México, no hay suficientes documentos históricos que den cuenta de cómo y cuales castigos escolares se han practicado a lo largo de la historia, A pesar de, hemos encontrado escritos que señalan que existieron dichos castigos con la finalidad de disciplinar y corregir, como se describe en la reglamentación del Liceo de varones, la escuela de Artes y Oficios y de Primeras Letras, como paseos por los salones y patios con un cartel pegado el pecho donde se escribía la causa del castigo, por ejemplo: burro, desaseado, etc. (Archivo Histórico de Jalisco, 1881- 1905 y 1920).

\subsubsection{Los castigos escolares y los derechos de la infancia y la adolescencia}

Por su parte, en el Estado Michoacán, desde el siglo XIX se emplearon medidas para regular los castigos, prohibido los azotes en todos los establecimientos de enseñanza en 1813, en cambio, preponderó la privación de la libertad, ya que se aceptaba el uso del calabozo junto con otras formas de castigo, como el detrimento de ciertos derechos, por ejemplo: no salir al recreo, no premios, etc. (Rondán, 2011).

Incluso, en el artículo 36 del Reglamento de Alumnos de la Escuela Nacional Preparatoria, expedido como decreto por Porfirio Díaz, Presidente Constitucional de los Estados Unidos Mexicanos, se acuerda que, los que no cumplan con alguna de las prevenciones de los Reglamentos escolares serán 
castigados por sus superiores con una amonestación privada o pública, o con encierro en el calabozo, según sea el caso (Archivo Histórico de Jalisco, 1885).

Al día de hoy, los castigos escolares no son historia, como se puede corroborar en los reportajes emitidos por diversos medios de comunicación, donde la controversia social originada por la implementación de la disciplina da lugar a diversos debates, quedando desfasada la autoridad del profesor dentro del aula.

En lo que respecta a los derechos de las niñas y los niños, inicialmente, en el periodo del gobierno de la Revolución Industrial, mueve la conciencia por legislar y proteger a la infancia, esto a raíz de la explotación laboral que sufrían en los diferentes trabajos forzados, ya que, se aprovechaba su juventud como fuerza de trabajo por el energía de sus pequeños cuerpos, lo que representaban menor necesidad de alimento exigiéndoles tareas agotadoras. De ahí, la concepción de la niñez como de menor categoría que el adulto. Posteriormente, en el siglo XX, a partir de los estudios pioneros de Tardieu, Athol Jonson y Henry Kempe, se acuña el término científico Síndrome del niño golpeado y se inician los estudios y observaciones relativos a las consecuencias del maltrato infantil. Como consecuencia se abrió la consideración de los niños y las niñas como sujetos de derecho, lo cual procedió finalmente en la promulgación de la Convención de los Derechos de la Niñez, con el fin de dar una respuesta legal que tuviera incidencia en lo social para hacer frente al problema del maltrato infantil (Sánchez, 2009). Por lo tanto, cuando el castigo se practicó en espacios públicos o privados se convirtió en un foco de atención para su regulación (Ossa, 2010).

Si bien, los correctivos han sido utilizados en todos los espacios escolares pese a lo expuesto, consideremos que un punto para la discusión es que los bordes son imprecisos, es decir, hasta qué punto terminan los buenos tratos, la buena educación, la disciplina y dónde comienza la indisciplina.

Consideramos, que un conflicto es la falta de una definición precisa que distinga entre la violencia escolar y disciplina escolar, esta situación ha llamado la atención de los derechos humanos y la salud pública, pues hace apenas unas décadas que se manifestó el interés del gobierno y la sociedad civil por este fenómeno en los niños, las niñas y los adolescentes, así como el reconocimiento de la necesidad de intervenir con programas y proyectos para la prevención en todos los niveles educativos (Saucedo, 2011).

Puntualmente, en América Latina la protección de la infancia es un tema que se ha tratado a lo largo del siglo XX. La Convención Sobre los Derechos del Niño en 1990, a un año de su aprobación por parte de la Asamblea General de la ONU, se propuso en la implementación de dicho tratado internacional, la creación los Códigos de la Niñez y adolescencia que, entre 1990 y 2009, se fueron dictando en la mayoría de los países de la región. Los que adquirieron cierta relevancia fueron los códigos de la niñez, cuya presencia data en fechas anteriores a la aprobación de la declaración de Ginebra de 1924. A partir de 
la aprobación por la Sociedad de Naciones de la denominada «Declaración de Ginebra» en 1924 se realizaron una serie de códigos relacionados a los derechos del niño. (Del Barrio et. al, 2003).

Concretamente en México se creó la Ley para la Protección de los Derechos de Niñas, Niños y Adolescente. Posteriormente, la mayoría de los países, excepto Argentina, Guatemala y Panamá recogen los códigos relacionados con la disciplina escolar que, según la $\mathrm{CDN}$, deberá ser compatible con la dignidad humana del niño. De esta forma, con mayor o menor precisión los códigos señalan el sentido de las medidas disciplinarias, prohibiendo el abuso y maltrato físico y psicológico y cualquier forma de castigo cruel y degradante (Dávila y Naya. 2003).

Por consiguiente, en México se creó la Ley para la protección de los derechos humanos de las niñas, los niños y adolescentes. La reforma se realizó en agosto del 2010, precisamente en el artículo 32 se especifica que las niñas, niños y adolescentes tienen derecho a una educación que respete su dignidad y les prepare para la vida en un espíritu de comprensión, paz y tolerancia. Y, en el artículo 3ro de la Constitución, se plantea: Se le proporcione atención, evitar la discriminación, se les impulse a la enseñanza y respeto de los derechos humanos y la convivencia sin violencia, no imponer medidas de disciplina que no estén previamente establecidas, sean contrarias a su dignidad, atenten contra su vida, su integridad física o mental (Ley para la protección de los derechos de las niñas, niños y adolescentes. Cámara de diputados del H. Congreso de la unión. 2010).

Que no sea una sociedad donde violentar los derechos humanos es un asunto cotidiano, violar los derechos y la dignidad de los alumnos parece algo natural; debemos pugnar por una humanidad más justa, democrática y educada, en done el respeto a la dignidad infantil sea conducta lógica y en la escuela (Moreno, 2010).

Navarro (2004. p.69). Expuso que la disciplina juega un papel preponderante en las instituciones educativas, no obstante. No se justifica el uso de la fuerza física y/o maltrato psicológico para mantener el orden en las aulas, también, manifestó que a atención no debe exigirse por autoridad, ni obtenerse por castigos, las amenazas nunca deben tomarse como medios disciplinarios dentro de las escuelas.

A pesar de, los malos comportamientos de los estudiantes reflejan la poca práctica de valores, razones, estas que no permiten el buen desenvolvimiento de la enseñanza-aprendizaje, por los problemas que se presentan. (Toala 2020)

\section{CONCLUSIONES}

Consideramos que la disciplina es un herramienta esencial en el proceso enseñanza-aprendizaje, asimismo, es significativo identificar y considerar la postura del estudiante, dicho de otro modo, estudiar su conducta dentro de la escuela. Es necesaria una normatividad sin ambigüedades en las instituciones 
educativas que establezca las formas adecuadas de implementar la disciplina, ya que esta problemática ha pasado de tener una connotación individual a una escala social que repercute en el espacio educativo, preocupando a la sociedad y a las autoridades educativas. Es importante que la enseñanza se base en los valores éticos y morales del individuo y sea un agente socializador en la escuela.

Recapacitamos que el decreto de los derechos de los niños, las niñas y adolescentes debería complementarse con un precepto que contemple los derechos de los profesores, ya que, la legislación ha rebasado la problemática escolar, se ha perdido el respeto a las autoridades escolares, por tal razón, el maestro es amedrentado por los estudiantes. El profesor está cada día más desprotegido ante las habituales discrepancias con sus alumnos.

Por último, diremos que se ha llegado a los límites donde lo esencial, lo básico, no es la formación académica, sino mantener el orden en las aulas, y así, no es posible llevar a cabo una enseñanza integral y la indisciplina está presente en los centros educativos del país, por tal razón, se deben tomar medidas para intervenir en los temas que perjudican el desarrollo curricular. 


\section{REFERENCIAS}

Archivo Histórico de Jalisco Instrucción Pública. [Material manuscrito]. Reglamento del Liceo de varones (IP caja 4) Archivo Histórico de Jalisco, Guadalajara, Jalisco, México. 1883.

Archivo Histórico de Jalisco Legislación. [Material impreso]. Decreto Presidencial Porfirio Díaz (IP caja 8) Archivo Histórico de Jalisco, Guadalajara, Jalisco, México. 1885.

Archivo Histórico de Jalisco Proyecto de reglamento de la Escuela de Artes y oficios del estado. [Material impreso]. (Instrucción Pública, caja 8) Archivo Histórico de Jalisco, Guadalajara, Jalisco, México. 1905.

Archivo Histórico de Jalisco Instrucción Pública. [Material manuscrito firmado por Gabino, Aceves]. Reglamento del Liceo de varones (IP caja 4) Archivo Histórico de Jalisco, Guadalajara, Jalisco, México. 1920

Dávila, P., Luis, M. \& Naya, N. Infancia, educación y códigos de la niñez en América latina. Revista española de educación comparad. 2010; 16, 213-233

Del Barrio CM., Martín, EO., Almeida, AM., \& Barrios, FA. Del maltrato y otros conceptos relacionados con la agresión entre escolares, y su estudio psicológico. Revista Infancia y aprendizaje. 2003; 26 (1): 924. Disponible en: http://docerver.ingentaconnect.com/deliver.

Furlan, A. Entre las "buenas intenciones" y los "acuerdos funcionales" Revista mexicana de investigación educativa 2005; (10): 27. 1083-1108

García, CA. La disciplina escolar. Murcia. Universidad de Murcia, Servicio de publicaciones, 2008; 134. Disponible en: http://www.um.es/publicaciones/digital/pdfs/disciplina.

Ley para la protección de los derechos de las niñas, niños y adolescentes. Cámara de diputados del H. Congreso de la unión. Secretaria General, secretaria de servicios parlamentarios, centro de documentación información y análisis. Última reforma 19-08-2010.

López, J. La disciplina escolar. Innovación y experiencias didácticas educativas. Revisita digital 2010; 33, 45: 1-8. Disponible en: http://www.csi-csif.es/andalucia/modules/mod_ense/revista/pdf/Numero

Márquez, GJ., Díaz, NJ., \& Cazzato, DS. La disciplina escolar: aportes de las teorías psicológicas. Revista de Artes y Humanidades UNICA 2007; 8 (18): 126-148. Recuperado en: http://redalyc.uaemex.mx/rdalyc/pdf/170/

Moreno, CM. Los castigos corporales en la escuela, una historia que no termina. Revista de educación y cultura, de la sección 47 del SNTE 1993; 2 (3): 8-9.

Navarro, EJ. Escuelas y castigos en niños y adolescentes. Anuario de filosofía, psicología y sociología. Universidad de Murcia y de la UNED de Cartagena 2004; (7): 67-94. Disponible en: http://dialnet.unirioja.es/servlet/articulo?codigo=1184598

OSSA, AF. El castigo como práctica de gobierno en la Reforma instruccioncita en Antioquia. Revista latinoamericana de ciencias sociales y niñez.2010; 8 (2): 1159 - 1168. Disponible en: http://www.umanizales.edu.co/revistacinde/index.html

Paima. Pepa El castigo corporal a niños es usual en América. Radio Nederland Wereloomredp Latinoamérica Publicado el 16 de septiembre a las 2:38 por redacción Informa RN. 2009. 
Quiñónez-Ortiz E. y Gómez-Pinillo L. (2020). La indisciplina y sus factores en la enseñanza $\begin{array}{llll}\text { aprendizaje. } M L S & \text { Psychology 21-38. }\end{array}$ https://doi.org/https://doi.org/10.33000/mlspr.v2i2.392

Roldán, RE. El castigo físico en la cultura escolar mexicana hacia mediados del siglo XIX. Conferencia dictada en el XI congreso Nacional de Investigación Educativa. Universidad Autónoma de México. 2011 disponible

http://www.academia.edu/1115114/El_Castigo_f\%C3\%ADsico_en_la_cultura_escolar_mexicana_hacia _mediados_de 1_siglo_XIX

Sánchez, GG. Teorías de niñas y niños sobre el castigo parental. Aportes para la educación y la crianza. Revista Electrónica Actualidades Investigativas en Educación 2009;9:(2):1-29 Recuperado en http://latindex.ucr.ac./ale-2009-2/al.

Saucedo, G. Reflexiones sobre la relación violencia de género y violencia escolar. Defensor. Comisión de Derechos humanos del Distrito federal. Revista de derechos humanos 2011; IX (9):25-26. Disponible en: http://dfensor.cdhdf.org.mx/DFensor_o9_20

Suárez, PM. Los castigos y otras estrategias disciplinarias vistos a través de los recuerdos escolares. Revista de educación 2004; (335): 429-443. Recuperado en http://www.revistaeducacion.mec.es/re335/.

Tovar, KE. El perfil del historiador: un análisis en la metodología de la investigación histórica. Revista estudiantil aula. Universidad de Cartagena, facultad de ciencias humanas 2014; (2): 86-93.

Treviño-Villarreal D. y. González- Medina M. (2020). Involucramiento docente y condiciones del aula: una díada para mejorar la convivencia escolar en bachillerato. Revista de Investigación Educativa, 38(2), 397-414. DOI:http://dx.doi.org/10.6018/rie.372241

Viana, S. (2010) Indisciplina en estudiantes de una escuela secundaria de Valladolid, Yucatán. Universidad Autónoma de Yucatán. Tesis de maestría no publicada, Universidad Autónoma de Yucatán. Yucatán, México. Libro 2015. Sesion_5_Concepcion_Chavez.pdf (inee.edu.mx) 